\title{
Performance of radial distribution function-based descriptors in the chemoinformatic studies of HIV-1 protease
}

\author{
Jurica Novak*,1,2, Maria A Grishina¹, Vladimir A Potemkin \& Johann Gasteiger ${ }^{3}$ \\ ${ }^{1}$ Higher Medical and Biological School, Laboratory of Computational Modeling of Drugs, South Ural State University, 20-A, \\ Tchaikovsky Str., Chelyabinsk, 454080, Russian Federation \\ ${ }^{2}$ Division of Physical Chemistry, Ruder Bošković Institute, Bijenička cesta 54, Zagreb, 10000, Croatia \\ ${ }^{3}$ Computer-Chemie-Centrum, Friedrich-Alexander-University Erlangen-Nürnberg, Naegelsbachstr. 25, 91052, Erlangen, Germany \\ *Author for correspondence: novaki@susu.ru
}

\begin{abstract}
Aim: This letter investigates the role of radial distribution function-based descriptors for in silico design of new drugs. Methodology: The multiple linear regression models for HIV-1 protease and its complexes with a series of inhibitors were constructed. A detailed analysis of major atomic contributions to the radial distribution function descriptor weighted by the number of valence shell electrons identified residues Arg8, Asp29 and residues of the catalytic triad as crucial for the correlation with the inhibition constant, together with residues Asp30 and lle50, whose mutations are known to cause an emergence of drug resistant variants. Conclusion: This study demonstrates an easy and fast assessment of the activity of potential drugs and the derivation of structural information of their complexes with the receptor or enzyme.
\end{abstract}

\section{Graphical abstract:}

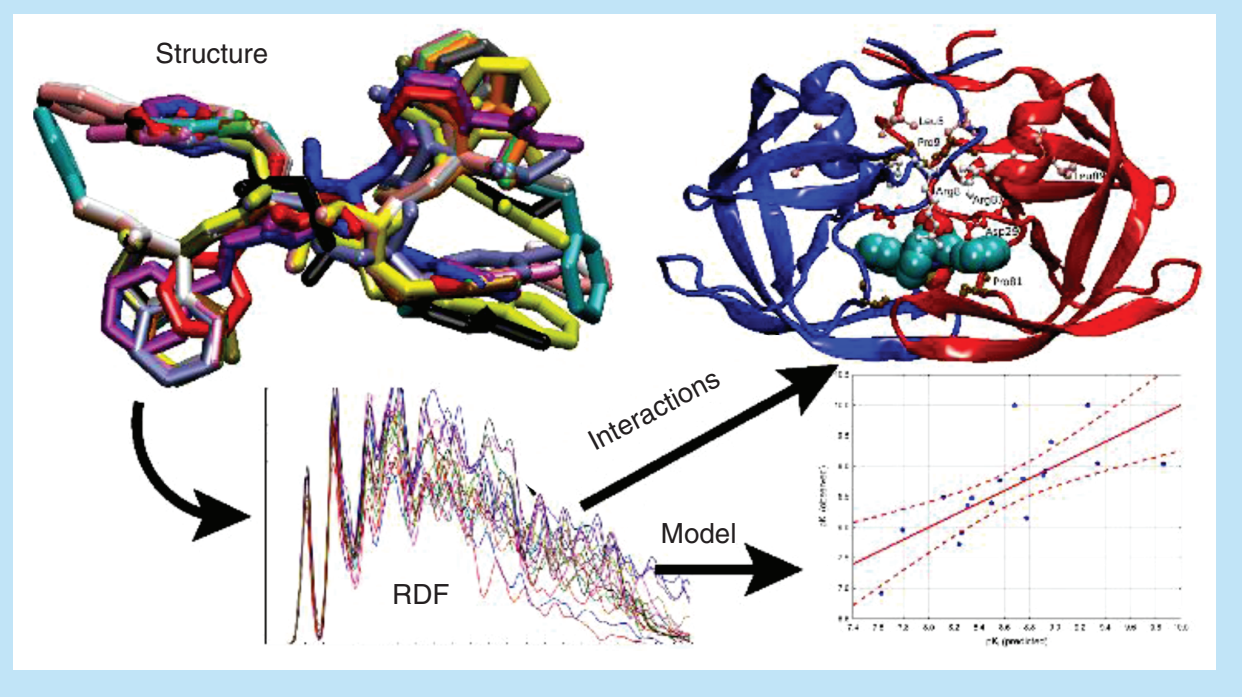

First draft submitted: 22 August 2019; Accepted for publication: 25 October 2019; Published online: 27 January 2020

Keywords: drug design $\bullet$ HIV protease $\bullet$ inhibitors $\bullet$ QSAR $\bullet$ radial distribution function $\bullet$ RDF

Highly active antiretroviral therapy is currently the most effective AIDS therapy. The combination of HIV protease inhibitors, reverse transcriptase inhibitors and/or an integrase inhibitors suppresses HIV replication and has reduced AIDS-related mortality sharply [1]. But the dark side of a lifelong treatment includes different patterns of unexpected systemic complications involving heart, bone, kidney and other organs [2-4]. Additionally, under continuous drug 
selective pressure, resistant forms of HIV-1 proteases were isolated [5], where a small number of point mutations at the protein pocket changes the interaction patterns between an enzyme and the drug, weakening the inhibitor's binding potential [6]. Studies of HIV protease mutants L90M, G48V and L90M/G48V showed that its affinity to drug saquinavir compared with the wild-type is reduced significantly [7]. Although the L90M mutation is not close to the active site, it most probably introduces perturbations with long-range structural consequences enhancing the catalytic activity of the mutant.

Maybe the best approach to prevent an emergence of a drug resistant virus is by suppressing viral replication. Tremendous efforts of the scientific community have been put into the search for appropriate chemical inhibitors to deactivate the HIV-1 protease [8-15], since its inhibition results in the production of immature, noninfectious viral particles. The HIV-1 protease, a homodimeric aspartyl protease, in its active form cleaves the peptide bond of a viral Gag and Gag-Pol polyprotein precursor into individual mature proteins [16]. In the active site two catalytic aspartic acid residues, one from each chain, meet at the dimer interface [17]. Two flexible $\beta$-hairpin flaps undergo a conformational shift upon substrate binding in the active site partially covering it. The natural substrate binds by establishing a complex interaction network between a minimum of seven amino acid residues and the enzymatic subsites $[18,19]$. The highly specific preference of HIV protease toward substrates also originates from the preferences of subsites for special types of side chains it accommodates. The $S 1, S 1^{\prime}, S 3$ and $S 3^{\prime}$ subsites prefer hydrophobic amino acids, while $\mathrm{S} 2$ and $\mathrm{S} 2$ ' accommodate both polar and hydrophobic residues [20].

According to the WHO, $59 \%$ of 36.9 million people living with HIV were receiving antiretroviral treatment by the end of 2017 [21]. Considering a myriad of drugs' side effects, viruses' high tendency to mutate and develop drug resistance, and the fact that the last time a HIV protease inhibitor was approved by US FDA in 2006 [22], the design of new protease inhibitors in subnanomolar regime and ideally without side reactions is of paramount importance. In recent years, numerous quantitative structure-activity relationship (QSAR) studies have appeared, trying to connect the structural characteristics of the inhibitor - HIV-1 protease complexes with the inhibition constant and ADMET properties [14,23-29]. Amin et al. have statistically validated different modeling approaches (Bayesian classification, HQSAR, 3D-QSAR CoMFA and CoMSIA analyses). Besides identifying the four regions of the hydroxyethylamine scaffold suitable for substitution with the primary aim of designing better inhibitors, they explore the influence of myriad substituents and identified crucial structural features of substituents responsible for increasing HIV-1 protease inhibition [14]. The 3D-QSAR model of thiazolidinone derivatives constructed by Ravichandran et al. [23] using CoMFA and CoMSIA computational models pointed out that the hydrogen bond acceptor and electrostatic parts have the largest contribution to the activity. Yoshida et al. combined molecular dynamics simulations of cyclic urea inhibitors - HIV-1 protease complexes with the fragment molecular orbital method to describe electronic features of the 3D structure of complexes. They introduced new electronic descriptors for both inhibitors and amino acid residues [24].

There are several QSAR studies involving radial distribution function (RDF) approach to correlate descriptors and the activity [30-34]. The model of Gonzales et al., based on the property weighted RDFs (weighted by atomic masses, by van der Waals volumes, by Sanderson electronegativities, and by atomic polarizabilities) and the optimum Bayesian regularized neural network model, is able to explain $87 \%$ of inhibitory activity variances for a set of 78 thiol and nonthiol peptidomimetic inhibitors of the farnesyltransferase enzyme [30]. Pingaew et al. correlated cytotoxic activities of a small set of 14 (thio) urea derivatives with a series of descriptors, with the conclusion that derivatives having high values of the RDF weighted by atomic van der Waals volumes display higher cytotoxicity against A549 cells [33]. The weighted RDF descriptors are most significant parameters for describing antimicrobial activity of the indenopyrazoles, according to the QSAR study of Mor et al. [34]

In this proof of concept study, we present a new strategy based on the (RDF, structural property) weighted by the number of outer shell electrons (electronic property) to correlate an easy-to-calculate structural descriptor with the inhibition constant or any activity related property of interest. The selection of the structures of the complexes was carried out to assess the subtle effects of interaction in very similar structures and to minimize the influence of the environment in the crystal due to the packing, observed in various space groups. In addition, our method shows the prospects for the study of large datasets of proteins in real time. This approach allows the evaluation of the bioactivity of newly designed drugs before synthesis and biological tests, saving a lot of time and avoiding expensive procedures. 


\section{Results \& discussion}

The similarity of structures of HIV type 1 protease, as well as a series of inhibitors and HIV-1 protease-inhibitor complexes have been examined adopting property-weighted RDFs.

$$
g(r)=\sum_{i=1}^{N-1} \sum_{j>i}^{N} p_{i} p_{j} e^{-a_{i j}^{-2 / 3}\left(r_{i j}-r\right)^{2}}
$$

Introduced by Hemmer et al. [35], $a_{\mathrm{ij}}$ is the sum of atomic polarizabilities of atoms $i$ and $j, r_{\mathrm{ij}}$ the distance between atoms $i$ and $j, N$ is the number of atoms in a molecule and the pre-exponential factors $p_{i}$ and $p_{j}$ account for the number of outer electrons of the $i^{\text {th }}$ and $j^{\text {th }}$ atoms, correspondingly. Atomic polarizabilities are calculated within the MERA model [36-38]. Atomic polarizabilities are taken by the degree of $-2 / 3$ to obtain a dimensionless value in the exponent (the unit of polarizability is $\AA^{3}$, therefore $a_{i j}^{-2 / 3}\left(r_{i j}-r\right)^{2}$ is a dimensionless value). Property-weighted RDFs with $p_{i}$ and $p_{j}$ have been found to be sensitive to the changes in enzymatic structure, needed for the estimation of the similarity of proteins and their complexes, and consequently for $\mathrm{pK}_{\mathrm{i}}$.

Each structure is represented by a vector, whose elements are calculated RDF values for a set of discrete points in space spanned by two atoms farthest apart, which is complex dependent and is in range between 57 and $60 \AA$, in intervals of $0.1 \AA$. The similarity, $\sigma_{\mathrm{ij}}$, was estimated for each pair of $i^{\text {th }}$ and $j^{\text {th }}$ structures using the following equations:

$$
\begin{gathered}
\sigma_{i j}=\frac{\int_{0}^{R_{e n z}} g_{i j}^{\min }(r) d r}{\int_{0}^{R_{e n z}} g_{i j}^{\text {mean }}(r) d r} \\
g_{i j}^{\text {min }}(r)=\min \left(g_{i}(r), g_{j}(r)\right) \\
g_{i j}^{\text {mean }}(r)=\frac{1}{2}\left(g_{i}(r)+g_{j}(r)\right)
\end{gathered}
$$

In other words, the similarity of structures $i$ and $j$ is the overlapped area divided by the mean area of the two RDFs. The fact that RDF is independent of the size of a molecule, that it unambiguously describes the $3 \mathrm{D}$ arrangement of the atoms, and that it is invariant against translation and rotation of a molecule, makes it a perfect candidate as a structure related descriptor in investigation of the relationship between structure and relevant properties in drug design, like the inhibition constant $\left(\mathrm{K}_{\mathrm{i}}\right)$ or half maximum effective concentration $\left(\mathrm{EC}_{50}\right)$ values. This is supported by previous studies, demonstrating that RDF descriptors outperform other methods, like constitutional, molecular walks counts, Galvez topological charge indices, 2D autocorrelations, Randić molecular profiles, geometrical, 3DMORSE and WHIM [39,40]. Finally, the multiple linear regression technique with the forward stepwise method is used to build a model for correlating the RDF at specific distances with the negative logarithm of the $\mathrm{K}_{\mathrm{i}}$ or with the $\mathrm{EC}_{50}$ values. Besides creating classical QSAR models, in this study, we present relevant residues identified as responsible for the correlation.

By querying the Protein Data Bank [41,42] for HIV-1 (EC = 3.4.23.16) structures with ligand and experimental inhibition constants, almost 300 structures were retrieved. To demonstrate the performance of newly introduced RDF-based descriptor, we opted for a smaller set, consisting of 18 structures of wild-type HIV-1 protease that crystallize in the P $22_{1} 2$ space group with different inhibitors bound in the catalytic pocket (see Supplementary Table 1), with published experimental inhibition constants. Although some water molecules might be hydrogen bonded to the atoms at the protein surface or even in the catalytic pocket, all crystallographic water molecules were discarded to reduce the random noise they might introduce. Our previous work on similar topic showed slightly worse results for model with included solvent molecules [37]. Then, RDFs were calculated.

The comparison of the primary structure of all wild-type proteins from our set showed that they have an identical sequence for both monomers. Only three structures (1W5V, 1W5W and 1W5X) differ. 12 extra amino acids before the protease sequence were added to them to facilitate the autocatalytic processing of the precursor protein during the expression procedure [43], but these were discarded from any further considerations. RDFs for all studied HIV-1 proteases without hydrogen atoms attached are very similar (PRO [protein]; Figure 1), sharing the same features, 


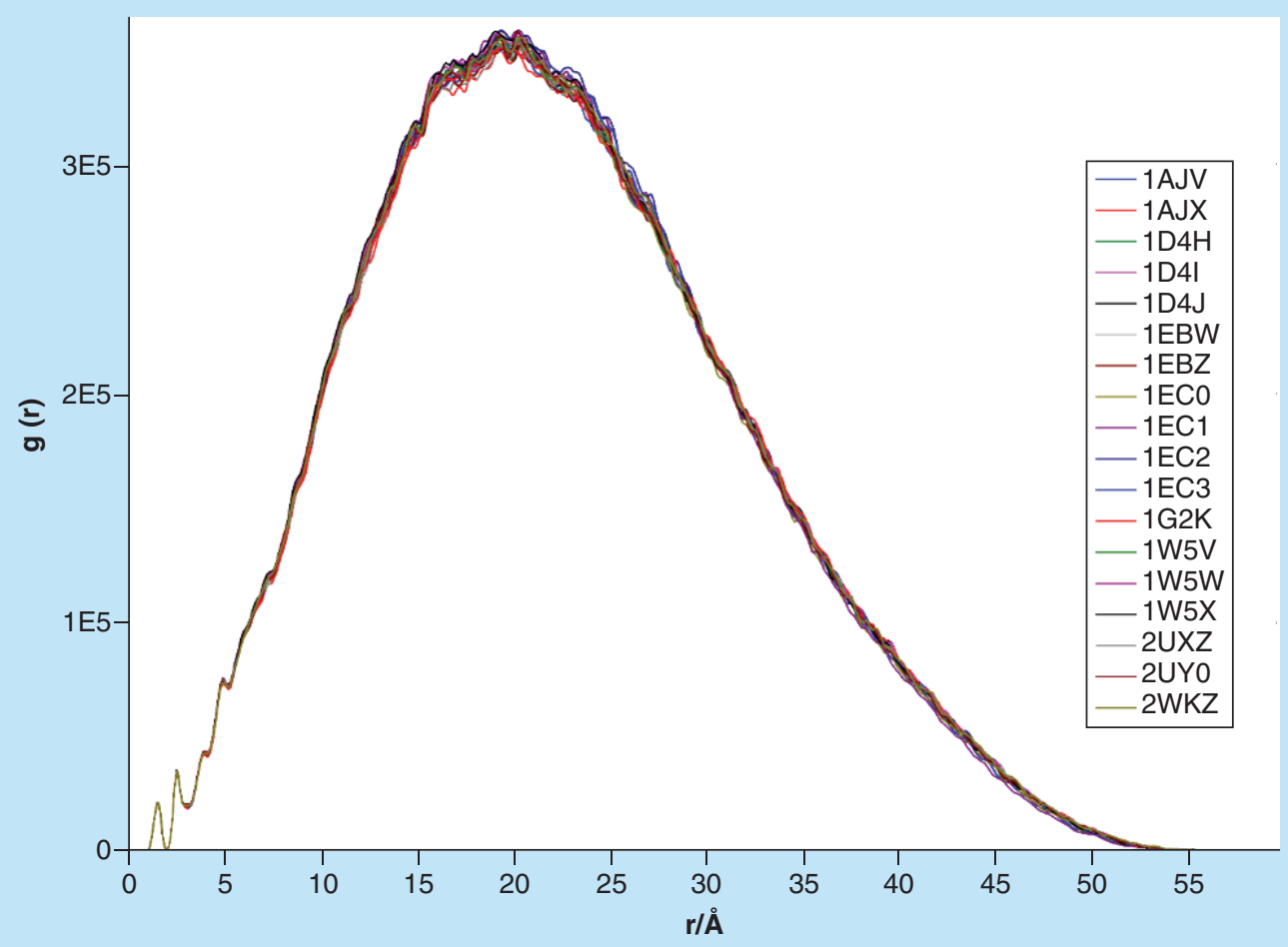

Figure 1. Radial distribution functions for HIV-1 proteases with only heavy atoms (protein).

like characteristic spikes below $6 \AA$, and global maxima between 18.9 and $20.2 \AA$. The comparison of the calculated RDFs revealed that the minimum similarity among the obtained similarities for the considered PDB structures of HIV-1 proteases without hydrogen atoms (PRO) is 0.951.

We begin our analysis by examining the minimum and maximum values of RDFs and its standard deviations for proteins without hydrogen atoms (Figures $1 \& 2$ ) The RDF values reach their maximum value at 19.3 and $19.2 \AA$, for the maximum and minimum RDF, respectively. For short distances, two well-separated peaks can be observed, followed by a shoulder just below $4 \AA$ and peak at $4.8 \AA$. For distances above $6 \AA$, details are lost and the shape of the RDF can be well approximated by the function:

$$
g(r) \approx a r^{2} e^{-b r^{2}}
$$

where the parameters $a$ and $b$ are determined by nonlinear regression. With the parameter $a$ in the range from 2160.1 to $2231.8 \AA^{-2}$ and the parameter $b$ between 0.00241 and $0.00247 \AA^{-2}$, this simple model explains $99.8 \%$ of the variance.

Proteins differ the most at a distance of $20.0 \AA$, where the standard deviation is 2887.5 (Figure 2). The standard deviation is $0.9 \%$ of the average RDF value for that distance (321482.8). That drives us to the conclusion that the $3 \mathrm{D}$ structure of the proteins is similar, with minor differences being a consequence of changes in the tertiary or quaternary structure of the protein, most probably induced by different orientations of the side chains of the residues near the surface of the protein.

At this point the real potency of the RDF-based descriptor is revealed. Although variations in the structure of the examined proteins are subtle, they are incorporated into the RDF descriptor and correlate very well with experimentally determined properties of the ligand-protein complex, such as $\mathrm{K}_{\mathrm{i}}$ and $\mathrm{EC}_{50}$.

Our regression model (Table 1) with only three descriptors for HIV-1 protease without hydrogen atoms (PRO), $\mathrm{g}_{16.1}, \mathrm{~g}_{20.5}$ and $\mathrm{g}_{22.6}$, is able to account for almost $73 \%$ of a variance in $\mathrm{pK}_{\mathrm{i}}$ values. To be more specific, for a pure 


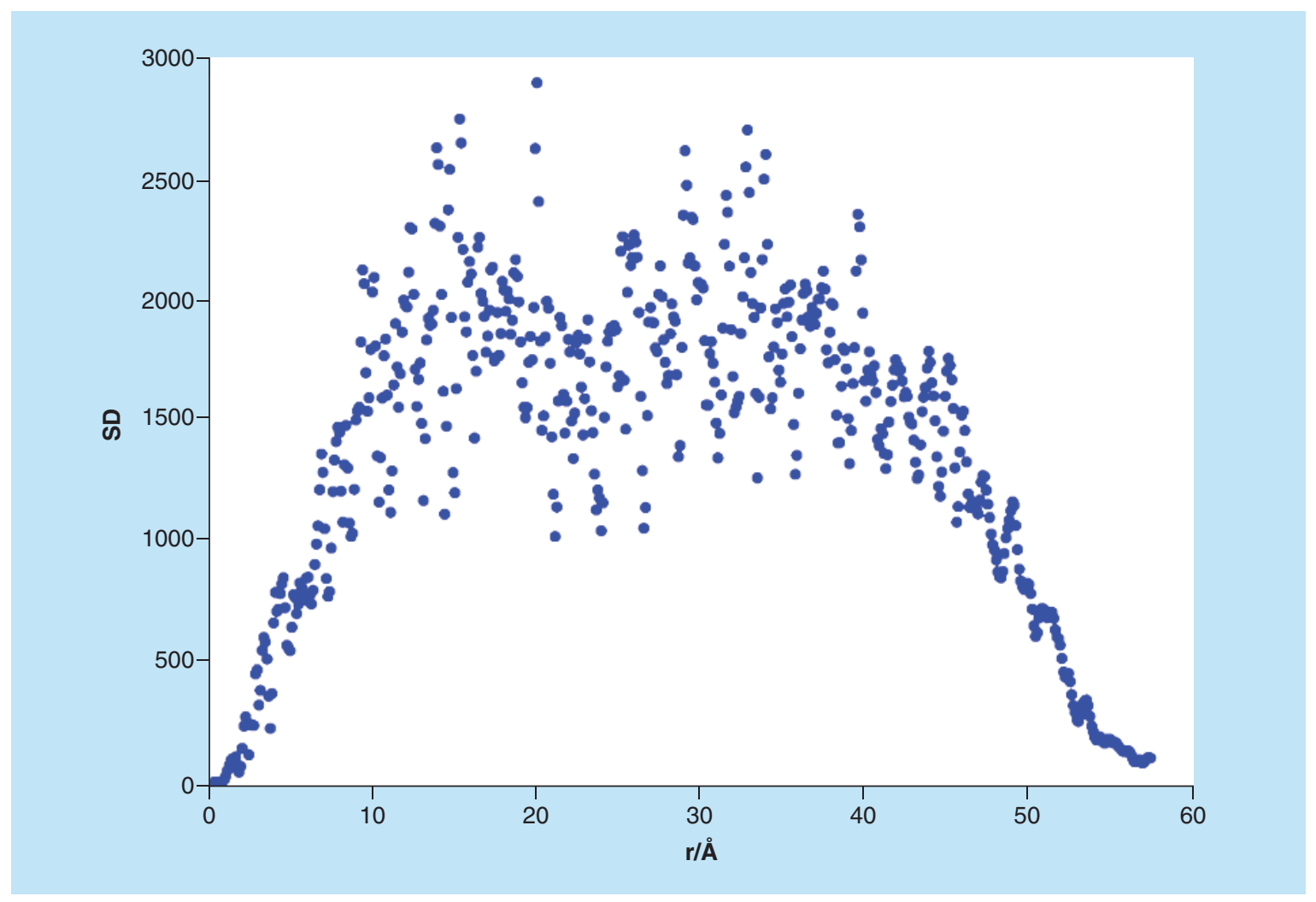

Figure 2. Standard deviations of radial distribution functions of a series of HIV-1 proteases without hydrogen atoms.

SD: Standard deviation.

\section{Table 1. Regression models relating radial distribution functions descriptors with $\mathrm{pK}_{\mathrm{i}}$ and $\mathrm{pEC} \mathrm{C}_{50}$.}

\begin{tabular}{|c|c|c|c|}
\hline System & QSAR model & $\mathbf{R}$ & SEoE \\
\hline PRO & $p K_{i}=74.663254699-0.000558759 g_{16.1}-0.000163396 g_{20.5}+0.000521181 g_{22.6}$ & 0.977 & 0.180 \\
\hline PRO & $p E C_{50}=17.806067614+0.001032702 g_{5.3}+0.000692144 g_{7.4}-0.000516745 g_{21.2}$ & 0.936 & 0.426 \\
\hline CPX & $p K_{i}=29.733712244-0.000716773 g_{3.3}-0.001089810 g_{49.6}+0.000752947 g_{50.0}$ & 0.880 & 0.401 \\
\hline CPX & $p E C_{50}=-68.351623342+0.001539991 g_{5.4}+0.000983847 g_{6.1}-0.000585298 g_{11.7}$ & 0.943 & 0.402 \\
\hline INH & $p K_{i}=8.550493244-0.005252941 g_{9.7}+0.006036735 g_{10.8}$ & 0.723 & 0.564 \\
\hline
\end{tabular}

CPX: Protein-inhibitor complex; $E_{50}$ : Half maximum effective concentration; INH: Inhibitor; $\mathrm{K}_{\mathrm{i}}$ : Inhibition constant; PRO: Protein; QSAR: Quantitative structure-activity relationship; R: Correlation coefficient; SEoE: Standard error of estimate.

protein the model equation describing the relationship between $\mathrm{pK}_{\mathrm{i}}$ values and $\mathrm{RDF}$ is:

$$
p K_{i}=74.663254699-0.000558759 g_{16.1}-0.000163396 g_{20.5}+0.000521181 g_{22.6}
$$

$g_{\mathrm{r}}$ is the value of the RDF descriptor at the distance $\mathrm{r}$. The correlation coefficient is 0.977 and the standard error of estimate 0.18 . Figure 3 presents the experimentally observed $\mathrm{pK}_{\mathrm{i}}$ values and those predicted by using equation (1). For the protein 1D4I the largest absolute residual is observed, with a difference between observed and calculated values of $\mathrm{pK}_{\mathrm{i}}$ just above the 0.3 margin (Supplementary Table 2).

More than 60 atoms from both chains provide a dominant contribution to the RDF at each of the three selected distances, where the dominant contribution is defined as an RDF value larger than the mean RDF contribution plus two standard deviations. If we compare atoms with dominant contribution to the RDFs for a distance at 16.1 $\AA$, one can see that there is a set of ten atom types common to both the A and $\mathrm{B}$ chains in every structure. Those atoms are part of the residues forming the protein pocket, where the substrate binds (Ala28, Asp25, Asp30 and 


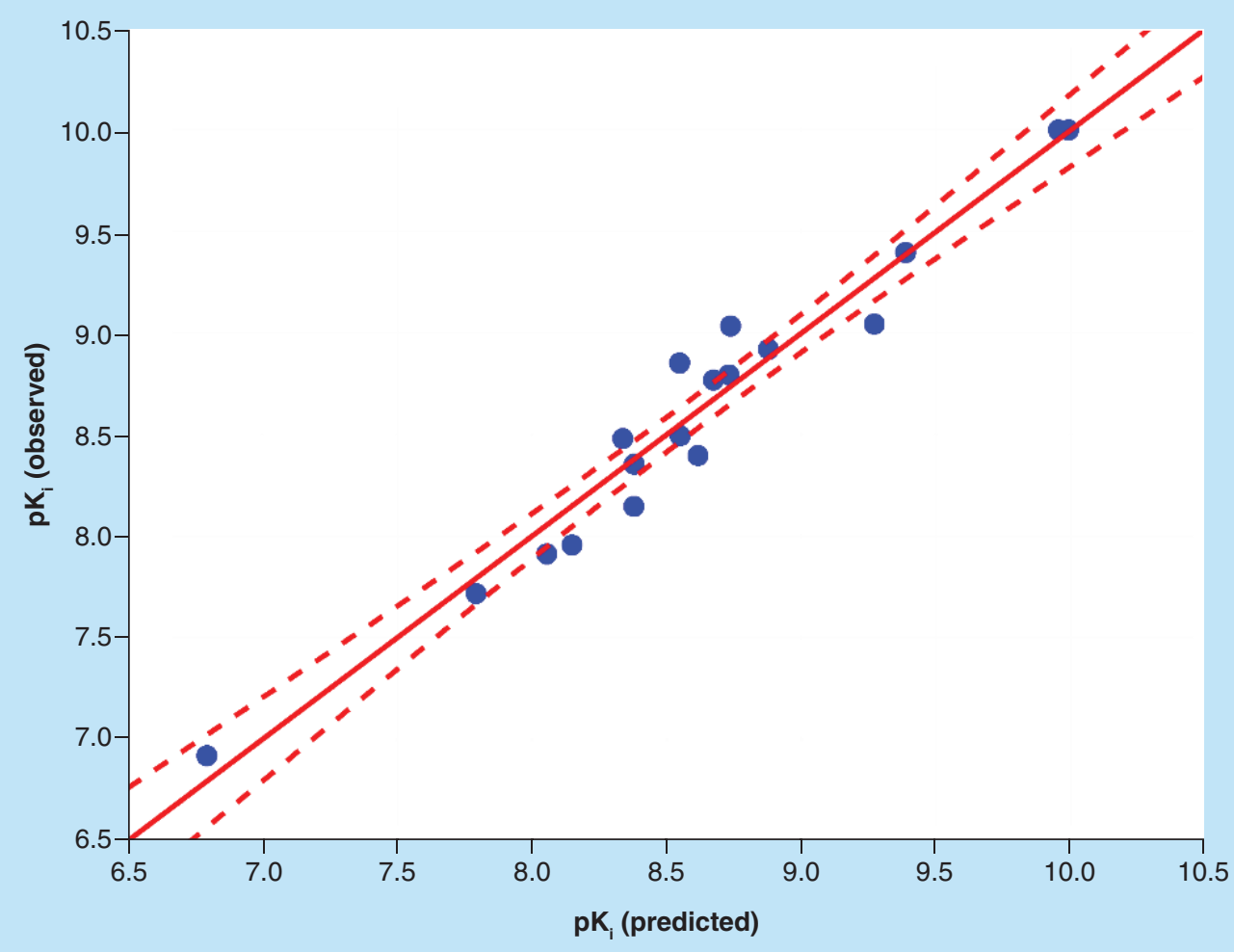

Figure 3. Experimentally observed versus predicted $\mathrm{pK}_{\mathrm{i}}$ values using equation (1), with $95 \% \mathrm{Cl}$ (dashed line).

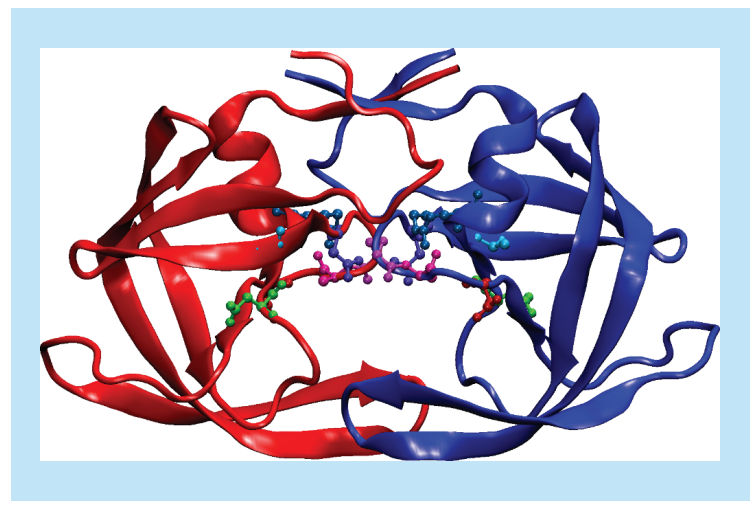

Figure 4. Residues with atoms having a dominant contribution to the $\mathrm{g}_{16.1}$ (CPK representation) shown for a structure of the HIV-1 protease. PDB ID: 1AJV.

Gly27), or is very near to it (Ala22, Asn83 and Arg87) (Figure 4). For example, both carboxylic oxygen atoms from the catalytic Asp25 are within the top five atoms with the most dominant RDF contribution by absolute value, revealing its paramount importance in catalytic activity. From the other side, the frequent occurrence of the oxygen atom of Ala28 and Asn83 forming a peptide bond highlights the importance of those residues in the neighboring parallel $\beta$ sheets as important structural factors responsible for protein stability.

The analysis of the atoms with dominant contributions to the descriptor $\mathrm{g}_{20.5}$ shows that they are positioned at the interface of the two subunits, like Thr26 and Gly27, completing all of the residues of the catalytic triad accounted within our model. Two oppositely charged residues, Arg8 and Asp29, have their side chains facing each other, influencing the quaternary structure, but at the same time can interact with the substrate, (de)stabilizing the substrate-enzyme complex. The atoms with dominant contribution to the descriptor $\mathrm{g}_{22.6}$ include the residues in direct contact to the solvent ( $\operatorname{Trp} 6, \mathrm{Gln} 7$ and Arg8) and the residues with potential to interact with the substrate (Arg8 and Ile50). This analysis exposed structurally and catalytically important residues without any a priori 
knowledge about it. The second confirmation of the validity of the suggested analysis are residues Asp30 and Ile50, which are both known to be important because their point mutations can induce clinically significant protease inhibitor resistance [44], although our model included only the wild-type HIV-1 protease.

An analogous statistical analysis was performed for the $\mathrm{pEC}_{50}$ values. Again, three RDF-based descriptors $\left(\mathrm{g}_{5.3}\right.$, $\mathrm{g}_{7.4}$ and $\mathrm{g}_{21.2}$ ) are enough to account for more than $87 \%$ of the variance for the PRO datasets (see Table 1 ). The dominant atoms include a variety of residues, from Gln58 pointing toward the solvent, Gly86 buried inside the enzyme, Cys95 on the interface of the two homodimers, to the conserved residues of the catalytic triad (Asp25Thr26-Gly27) and residues directly interacting with the substrate - Arg8, Leu23, Asp29 and Pro81. While Arg8 can form a salt bridge with Asp29 from the other chain [45], all four residues are close enough to interact with the P1 or P2 side chains of the substrate when bound, with distances of the heavy atoms varying between 3 and $5 \AA$. Leu23 and Pro81 are establishing a hydrophobic interaction with the P1 part of the substrate.

We would like to point out a few advantages of the RDF-based descriptors calculated directly from the PDB file adopted throughout this investigation, without any hydrogen atoms included. Since x-ray crystallography frequently cannot determine positions of hydrogen atoms because of their too small electron density, it is hard to tell in which protonation states residues' side chains are. The complexity and importance of the determination of protonation states has been demonstrated on HIV-1 protease [46-51]. Nam et al. [48] performed ab initio calculations of the active site of HIV-1 protease and used free energy perturbation method to determine the binding free energy of the neutral inhibitor (A74704) to four different protonation states of Asp25 and Asp25' residues. Their analysis corroborate that in case of a neutral inhibitor the enzyme is in mono-protonated state (the carboxylic group of Asp25 is neutral, while Asp25' has a deprotonated side chain). Other methods, like $\mathrm{pH}$ rate and solvent kinetic isotope effects studies [46], NMR studies of site specifically ${ }^{13} \mathrm{C}$ labeled $\mathrm{HIV}-1$ protease [47,50] and the molecular mechanics Poisson-Boltzmann surface area method combined with molecular dynamics simulations [49], confirmed the presence of both carboxylate and carboxyl moieties in the catalytic triad. Our approach with the calculation of RDFs for heavy atoms only is robust enough, making a calculation of the $\mathrm{pK}$ values of side chains and refinement of hydrogen positions obsolete, saving a lot of time.

In the second part of this investigation, the inhibitor is bound to the active site of HIV-1 protease. The main features of RDFs are similar to those without inhibitor (see Supplementary Figure 1), with global maxima between 18.9 and $20.3 \AA$, and the individual low-lying peaks at 1.5, 2.5, 3.9 and $4.8 \AA$. The largest standard deviation (4186.4) has moved to a greater distances $\left(24.8 \AA\right.$ ), being only $1.3 \%$ of the mean $g_{\mathrm{r}}$ value at that distance (Supplementary Figure 2). The introduction of an inhibitor does not significantly change the RDFs, only a miniature decrease in the RDFs similarity coefficient to 0.950 is observed for the protein-inhibitor complex (CPX) compared with 0.951 for PRO.

The linear regression analysis of the RDFs of inhibitor-protein complex without hydrogen atoms included $(\mathrm{CPX})$ resulted in a model connecting $\mathrm{pK}_{\mathrm{i}}$ values and three descriptors, $\mathrm{g}_{3.3}, \mathrm{~g}_{49.6}$ and $\mathrm{g}_{50.0}$

$$
p K_{i}=29.733712244-0.000716773 g_{3.3}-0.001089810 g_{49.6}+0.000752947 g_{50.0}
$$

with the correlation coefficient of 0.880 and standard error of estimate 0.40 .

It is interesting that atoms with the largest contributions to the $\mathrm{g}_{3.3}$ descriptor and that are present in all studied structures include three residues that are part of the $\alpha$ helix, the hydrophobic Val11 with the side chain oriented toward the $\alpha$ helix and oxygen atoms from the catalytic Asp25. At larger distances, the neighboring residues Pro39, Gly40 and Arg41 have more than three special atoms, with their side chains exposed to the solvent.

$$
p E C_{50}=-68.351623342+0.001539991 g_{5.4}+0.000983847 g_{6.1}-0.000585298 g_{11.7}
$$

Equation (2) describes the relationship between the negative logarithm of the $\mathrm{EC}_{50}$ value and the RDF descriptor for CPX, with a coefficient of correlation of 0.943 and a standard error of estimate of 0.40 . This model is capable of describing more than $88 \%$ of the total variance. As can be seen from Figure 5, atoms with dominant contributions to the RDF include all three types of residues - residues interacting with the substrate, residues at the interface between two subunits and the residues exposed to the solvent. At the interface of two homodimers is Thr26, a part of the conserved triad, and the polar Asn 98 at the top of the complex, being part of the antiparallel $\beta$ sheet. While positively charged Arg57 is in contact with the solvent, Val32 and Ile84 are close enough to the substrate to establish a hydrophobic interactions. 

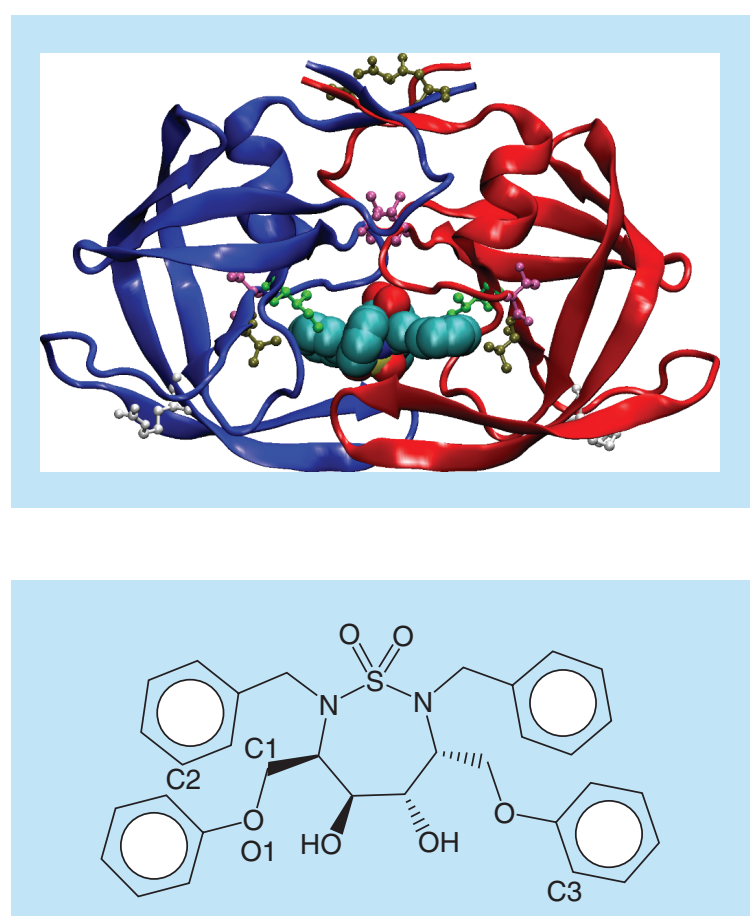

Figure 5. Residues with atoms with dominant contribution to the radial distribution function descriptors $g_{5.4}, g_{6.1}$ and $g_{11.7}$ (CPK representation) for CPX structures of the HIV-1 protease with inhibitor NMB (VdW representation). PDB ID: 1AJV.

Figure 6. Structure and numbering of atoms for inhibitor NMB.

Finally, the analysis of the RDFs of inhibitors alone was performed. The RDFs similarity coefficient is low, being only 0.631 for inhibitors without hydrogen atoms (INH). Although the structures of the inhibitors are very different, only two descriptors, $\mathrm{g}_{9.7}$ and $\mathrm{g}_{10.8}$, are necessary to correlate the $\mathrm{pK}_{\mathrm{i}}$ values with the inhibitors' RDFs in the model for INH, while the analysis of the dominant contributions to the RDF has been performed for each inhibitor separately.

According to the extensive study of the protein-ligand interactions for more than 11,000 complexes including over 750,000 ligand-protein atom pairs by Ferreira De Freitas and Schapira [52], the most frequent interactions include hydrophobic contacts, hydrogen bonds and aromatic interactions. Dominant atoms for $\mathrm{g}_{9.7}$ descriptor for inhibitor (2R,3R,4S,5S,6R,7R)-1,1-dioxo-3,6-bis(phenoxymethyl)-2,7-bis(phenylmethyl)-1,2,7-thiadiazepane-4,5diol (NMB) include $\mathrm{C} 1$ and $\mathrm{O} 1$ atoms (for numbering see Figure 6), with the distance to the heavy atoms of Gly27, Ala28 and Ile50 being below $4 \AA$. Atoms C2 and C3 have the largest contributions to the g 10.8 descriptor, establishing hydrophobic interactions with Leu23, and Gly27 oxygen atoms only $3.28 \AA$ away from them.

\section{Conclusion}

In this letter, we demonstrate the successful application of molecular descriptors based on a RDF to correlate it with biologically interesting properties of a ligand-enzyme complex, like $\mathrm{pK}_{\mathrm{i}}$ and $\mathrm{pEC}_{50}$. Although the difference between the studied structures and their RDFs are small, linear regression analysis enabled us to create models with only three easily calculated descriptors that account for more than $75 \%$ of the variance in the experimentally measured inhibition constants and the half-maximum effective concentration. There are two main advantages of the proposed approach. First, since only nonhydrogen atoms are being considered, there is no need to determine the protonation states of the side chains of the residues. The benefit is twofold - the error associated with wrong predictions of protonation states is avoided and time savings in man- and computer power, especially for larger sets, can be substantial. Second, simplicity and the unique features of the RDF make it easy to apply to any system. Once the model is built, only a small number of descriptors have to be calculated. Analysis of atoms with dominant contributions to the RDFs can be of interest for drug design to identify relevant atoms and interactions.

\section{Future perspective}

Machine learning, a subfield of artificial intelligence, has entered to the field of drug discovery. There are myriad examples of its successful application in various fields of modern chemistry, naming just the supervised learning methods in the QSAR studies used to predict different properties. With databases getting larger and larger, new, 
reliable and fast methods with high predictability potential are mandatory for the computational-aided drug design. Therefore, the results of this study may be incorporated in the next generation virtual screening methods and used as analyzing tool for recognition of relevant features and interactions.

\section{Summary points}

- The new radial distribution function (RDF) descriptor weighted by the number of valence shell electrons is introduced to describe ligand-enzyme complexes.

- The correlation between RDF based descriptor and physico-chemical properties is established.

- The analysis of atomic contribution to the total RDF can identify relevant residues and interactions.

- The unique features of the RDF make it easy to apply to any system.

\section{Supplementary data}

To view the supplementary data that accompany this paper please visit the journal website at: www.future-science.com/doi/suppl /10.4155/fmc-2019-0241

\section{Financial \& competing interests disclosure}

This work was supported by the Government of the Russian Federation (Act 211, contract 02.A03.21.0011) and by the Ministry of Science and Higher Education of the Russian Federation (grants 4.8298.2017/8.9, 4.7279.2017/8.9), Russian Foundation for Basic Research (grant 18-53-45015). The authors have no other relevant affiliations or financial involvement with any organization or entity with a financial interest in or financial conflict with the subject matter or materials discussed in the manuscript apart from those disclosed.

No writing assistance was utilized in the production of this manuscript.

\section{References}

Papers of special note have been highlighted as: $\bullet$ of interest

1. Wang Y, Lv Z, Chu Y. HIV protease inhibitors: a review of molecular selectivity and toxicity. HIV AIDS (Auckl.) 7, 95 (2015).

2. Kramer-Hämmerle S, Rothenaigner I, Wolff H, Bell JE, Brack-Werner R. Cells of the central nervous system as targets and reservoirs of the human immunodeficiency virus. Virus Res. 111(2), 194-213 (2005).

3. Palmisano L, Vella S. A brief history of antiretroviral therapy of HIV infection: success and challenges. Ann. Ist. Super. Sanita. 47(1), 44-48 (2011).

4. Maartens G, Celum C, Lewin SR. HIV infection: epidemiology, pathogenesis, treatment, and prevention. Lancet 384(9939), 258-271 (2014).

5. Pillay D, Taylor S, Richman DD. Incidence and impact of resistance against approved antiretroviral drugs. Rev. Med. Virol. 10(4), 231-253 (2000).

6. Condra JH, Schleif WA, Blahy OM et al. In vivo emergence of HIV-1 variants resistant to multiple protease inhibitors. Nature 374(6522), 569-571 (1995).

7. Maschera B, Darby G, Palu G, Wright L, Tisdale M. Mutations in the viral protease that confer resistance to saquinavir increase the dissociation rate constant of the protease saquinavir complex. J. Biol. Chem. 271(52), 33231-33235 (1996).

8. Schiffer Ca, Ali A, Reddy GSKK et al. Discovery of HIV-1 protease inhibitors with picomolar affinities incorporating $\mathrm{N}$-aryl-oxazolidinone-5-carboxamides as novel P2 ligands. J. Med. Chem. 49(25), 7342-7356 (2006).

9. Reddy GSKK, Ali A, Nalam MNL et al. Design and synthesis of HIV-1 protease inhibitors incorporating oxazolidinones as P2/P2' ligands in pseudosymmetric dipeptide isosteres. J. Med. Chem. 50(18), 4316-4328 (2007).

10. Altman MD, Ali A, Kumar Reddy GSK et al. HIV-1 protease inhibitors from inverse design in the substrate envelope exhibit subnanomolar binding to drug-resistant variants. J. Am. Chem. Soc. 130(19), 6099-6113 (2008).

11. Ali A, Reddy GSKK, Nalam MNL et al. Structure-based design, synthesis, and structure-activity relationship studies of HIV-1 protease inhibitors incorporating phenyloxazolidinones. J. Med. Chem. 53(21), 7699-7708 (2010).

12. Islam MA, Pillay TS. Simplified molecular input line entry system-based descriptors in QSAR modeling for HIV-protease inhibitors. Chemom. Intell. Lab. Syst. 153, 67-74 (2016).

13. Bhargava S, Adhikari N, Amin SA, Das K, Gayen S, Jha T. Hydroxyethylamine derivatives as HIV-1 protease inhibitors: a predictive QSAR modelling study based on Monte Carlo optimization. SAR QSAR Environ. Res. 28(12), 973-990 (2017).

14. Amin SA, Adhikari N, Bhargava S, Jha T, Gayen S. Structural exploration of hydroxyethylamines as HIV-1 protease inhibitors: new features identified. SAR QSAR Environ. Res. 29(5), 385-408 (2018). 
15. Halder AK. Finding the structural requirements of diverse HIV-1 protease inhibitors using multiple QSAR modelling for lead identification. SAR QSAR Environ. Res. 29(11), 911-933 (2018).

16. Wlodawer A, Vondrasek J. Inhibitors of HIV-1 protease: a major success of structure-assisted drug design. Annu. Rev. Biophys. Biomol. Struct. 27, 249-284 (1998).

17. Brik A, Wong CH. HIV-1 protease: mechanism and drug discovery. Org. Biomol. Chem. 1(1), 5-14 (2003).

18. Gustchina A, Sansom C, Prevost M et al. Energy calculations and analysis of HIV-1 protease-inhibitor crystal structures. Protein Eng. Des. Sel. 7(3), 309-316 (1994).

19. Ghosh AK, Chapsal BD. Design of the anti-HIV protease inhibitor darunavir. In: Introduction to Biological and Small Molecule Drug Research and Development: Theory and Case Studies. Ganellin C, Jefferis R, Roberts S. (Eds). Elsevier Ltd, Oxford, UK, 355-384 (2013).

20. Ghosh AK, Osswald HL, Prato G. Recent progress in the development of HIV-1 protease inhibitors for the treatment of HIV/AIDS. J. Med. Chem. 59(11), 5172-5208 (2016).

- Describes the development of the new generation HIV protease inhibitors.

21. World Health Organization (2019). www.who.int/hiv/data/en/

22. U.S. Food and Drug Administration (2019). www.fda.gov/forpatients/illness/hivaids/treatment/ucm118915.htm

23. Ravichandran V, Prashantha Kumar BR, Sankar S, Agrawal RK. Predicting anti-HIV activity of 1,3,4-thiazolidinone derivatives: 3D-QSAR approach. Eur. J. Med. Chem. 44(3), 1180-1187 (2009).

24. Yoshida T, Fujita T, Chuman H. Novel quantitative structure-activity studies of HIV-1 protease inhibitors of the cyclic urea type using descriptors derived from molecular dynamics and molecular orbital calculations. Curr. Comput. Aided-Drug Des. 5(1), 38-55 (2009).

25. Ravichandran V, Mourya VK, Agrawal RK. Prediction of HIV-1 protease inhibitory activity of 4-hydroxy-5,6-dihydropyran-2-ones: QSAR study. J. Enzyme Inhib. Med. Chem. 26(2), 288-294 (2011).

26. Saranya N, Selvaraj S. QSAR studies on HIV-1 protease inhibitors using non-linearly transformed descriptors. Curr. Comput. Aided-Drug Des. 8(1), 10-49 (2012).

27. Fatemi MH, Heidari A, Gharaghani S. QSAR prediction of HIV-1 protease inhibitory activities using docking derived molecular descriptors. J. Theor. Biol. 369, 13-22 (2015).

28. Tong J, Zhan P, Bai M, Yao T. Molecular modeling studies of human immunodeficiency virus type 1 protease inhibitors using three-dimensional quantitative structure-activity relationship, virtual screening, and docking simulations. J. Chemom. 30(9), 523-536 (2016).

29. Tong J, Wu Y, Bai M, Zhan P. 3D-QSAR and molecular docking studies on HIV protease inhibitors. J. Mol. Struct. 1129, 17-22 (2017).

30. González MP, Caballero J, Tundidor-Camba A, Helguera AM, Fernández M. Modeling of farnesyltransferase inhibition by some thiol and non-thiol peptidomimetic inhibitors using genetic neural networks and RDF approaches. Bioorganic Med. Chem. 14(1), 200-213 (2006).

31. González MP, Gándara Z, Fall Y, Gómez G. Radial distribution function descriptors for predicting affinity for vitamin D receptor. Eur. J. Med. Chem. 43(7), 1360-1365 (2008).

32. Hemmateenejad B, Javidnia K, Nematollahi M, Elyasi M. QSAR studies on the antiviral compounds of natural origin. J. Iran. Chem. Soc. 6(2), 420-435 (2009).

33. Pingaew R, Tongraung P, Worachartcheewan A et al. Cytotoxicity and QSAR study of (thio)ureas derived from phenylalkylamines and pyridylalkylamines. Med. Chem. Res. 22(8), 4016-4029 (2013).

34. Mor S, Mohil R, Nagoria $S$ et al. Regioselective synthesis, antimicrobial evaluation and QSAR studies of some 3-aryl-1-heteroarylindeno[1,2- c]pyrazol-4(1 H)-ones. J. Heterocycl. Chem. 54(2), 1327-1341 (2017).

35. Hemmer MC, Steinhauer V, Gasteiger J. Deriving the 3D structure of organic molecules from their infrared spectra. Vib. Spectrosc. 19(1), 151-164 (1999).

36. Potemkin VA, Bartashevich EV, Belik AV. A new approach to predicting the thermodynamic parameters of substances from molecular characteristics. Russ. J. Phys. Chem. 70(3), 411-416 (1996).

37. Potemkin VA, Pogrebnoy AA, Grishina MA. Technique for energy decomposition in the study of "receptor-ligand" complexes. J. Chem. Inf. Model. 49(6), 1389-1406 (2009).

38. Potemkin V, Potemkin A, Grishina M. Internet resources for drug discovery and design. Curr. Top. Med. Chem. 18(22), 1955-1975 (2019).

- An important review covering a variety of online resources used for drug discovery and design.

39. González MP, Terán C, Fall Y, Teijeira M, Besada P. A radial distribution function approach to predict A 2B agonist effect of adenosine analogues. Bioorganic Med. Chem. 13(3), 601-608 (2005).

40. Helguera AM, Cabrera Pérez MÁ, González M. A radial-distribution-function approach for predicting rodent carcinogenicity. J. Mol. Model. 12(6), 769-780 (2006).

41. Berman HM, Westbrook J, Feng Z et al. The Protein Data Bank. Nucleic Acids Res. 28(1), 235-242 (2000). 
42. RCSB PDB (2019). www.rcsb.org/

43. Lindberg J, Pyring D, Löwgren S et al. Symmetric fluoro-substituted diol-based HIV protease inhibitors: ortho-fluorinated and meta-fluorinated P1/P1'-benzyloxy side groups significantly improve the antiviral activity and preserve binding efficacy. Eur. J. Biochem. 271(22), 4594-4602 (2004).

44. HIV drug resistance database (2019). https://hivdb.stanford.edu/dr-summary/resistance-notes/PI/

45. Yamazaki T, Nicholson LK, Torchia DA et al. NMR and $\mathrm{x}$-ray evidence that the HIV protease catalytic aspartyl groups are protonated in the complex formed by the protease and a non-peptide cyclic urea-based inhibitor. J. Am. Chem. Soc. 116(23), 10791-10792 (1994).

46. Hyland LJ, Tomaszek TA, Meek TD. Human immunodeficiency virus-1 protease. 2. Use of pH rate studies and solvent kinetic isotope effects to elucidate details of chemical mechanism. Biochemistry 30(34), 8454-8463 (1991).

47. Wang YX, Freedberg DI, Yamazaki T et al. Solution NMR evidence that the HIV-1 protease catalytic aspartyl groups have different ionization states in the complex formed with the asymmetric drug KNI-272. Biochemistry 35(31), 9945-9950 (1996).

48. Nam KY, Chang BH, Han CK, Ahn SG, No KT. Investigation of the protonated state of HIV-1 protease active site. Bull. Korean Chem. Soc. 24(6), 817-823 (2003).

49. Chen J, Yang M, Hu G, Shi S, Yi C, Zhang Q. Insights into the functional role of protonation states in the HIV-1 protease-BEA369 complex: molecular dynamics simulations and free energy calculations. J. Mol. Model. 15(10), 1245-1252 (2009).

50. Torbeev VY, Kent SBH. Ionization state of the catalytic dyad Asp $25 / 25^{\prime}$ in the HIV-1 protease: NMR studies of site-specifically ${ }^{13} \mathrm{C}$ labelled HIV-1 protease prepared by total chemical synthesis. Org. Biomol. Chem. 10(30), 5887-5891 (2012).

51. Weber IT, Waltman MJ, Mustyakimov M et al. Joint x-ray/neutron crystallographic study of HIV-1 protease with clinical inhibitor amprenavir: insights for drug design. J. Med. Chem. 56(13), 5631-5635 (2013).

52. Ferreira De Freitas R, Schapira M. A systematic analysis of atomic protein-ligand interactions in the PDB. Med. Chem. Comm. 8(10), 1970-1981 (2017).

- A great insight into the nature of protein-ligand interactions. 